\title{
ЧИСЛЕННОЕ МОДЕЛИРОВАНИЕ И ЭКСПЕРИМЕНТАЛЬНОЕ ИССЛЕДОВАНИЕ ПРОЦЕССОВ ГИДРОДИНАМИКИ И ТЕПЛООБМЕНА В ТЕПЛООБМЕННЫХ АППАРАТАХ НОВОЙ КОНСТРУКЦИИ
}

\author{
Горобец В.Г. ${ }^{1}$, доктор техн. наук., Богдан Ю.А. ${ }^{2}$, Троханяк В.И. ${ }^{1}$ \\ ${ }^{1}$ Национальный университет биоресурсов и природоиспользования Украинь,, \\ ул. Героев Обороны, 15, Киев, 03041, Украина \\ ${ }^{2}$ Киевская государственная академия водного транспорта имени гетмана Петра Конашевича- \\ Сагайдачного, ул. Кирилловская, 9, Киев, 04071, Украина
}

\begin{tabular}{|c|c|}
\hline \multicolumn{2}{|l|}{ Проведено } \\
\hline експерименталь- & рование и экспериментальное ис- \\
\hline дослідження & и гидроди- \\
\hline гідродинамічних & намических характеристик сжатых \\
\hline компактних пучків круглих труб & пучков круглых труб в теплообмен- \\
\hline апаратах нової & овой конструкции. \\
\hline конструкції. Порівняння результатів & Сравнение результатов численного \\
\hline моделювання стисне- & моделирования сжатых пучков кру- \\
\hline них пучків круглих труб з резуль- & результатами проведен- \\
\hline татами проведеного експерименту & римента и известными \\
\hline та відомими експериментальними & экспериментальными данными для \\
\hline даними для пучків круглих труб & пучков круглых труб традиционной \\
\hline традиційної & коридорной компоновки, подтверж- \\
\hline підтверджує & дает адекватность моделирования \\
\hline моделювання i майже & и почти двойной рост тепловой эф- \\
\hline зростання & фективности исследуемых сжатых \\
\hline досліджуваних стиснених пучків & пучков труб при незначительных \\
\hline труб при не & отличиях суммарного аэродинами- \\
\hline сумар & \\
\hline
\end{tabular}

Numerical modeling and experimental investigation of heat and hydrodynamic characteristics of compressed round tube bundle for heat exchangers of new design are presented. Comparison of the results of numerical modeling of compressed round tube bundle with results of finished experiment and knowing experimental data for round tube bundle of convention inline geometry, confirms the adequacy of modeling and almost double the increase in the thermal efficiency of the investigated compressed round tube bundle with a relatively small change in the total aerodynamic resistance.

Библ.12. рис. 7.

Ключевые слова: вакуум-выпарной аппарат, тепловой насос, оптимизация.

$\alpha$ - коэффициент теплоотдачи, Вт/ $\mathrm{M}^{2} \mathrm{~K}$;

$d$ - внешний диаметр трубы, м;

$\delta$ - толщина стенки трубы, м;

$H$ - площадь поверхности теплообмена (суммарная для двух тепловоспринимающих участков поверхность нагрева), м $^{2}$;

$l$ - длина трубы, м;

$D$ - диаметр, м;

$G$ - расход, кг/с;

$T$ - температура, $\mathrm{K},{ }^{\circ} \mathrm{C}$;

$P$ - давление, Па;

$S$ - шаг размещения труб в пучке, м;

$i$ - количество труб в ряду пучка;

$j$ - количество рядов в пучке; $z$ - общее количество труб в пучке;

$k$ - кинетическая энергия турбулентности;

$\varepsilon-$ скорость диссипации турбулентности;

$w$ - скорость потока теплоносителя, м/с;

$\tau$ - время, с;

$x, y$ - декартовые координаты;

$t$ - температура, $\mathrm{K},{ }^{\circ} \mathrm{C}$;

$n$ - частота вращения коленчатого вала, мин $^{-1}$;

$\varphi$ - угол по окружности трубы, ${ }^{\circ}$;

$c$ - удельная теплоемкость, Дж/(кг·К);

$a$ - относительный поперечный шаг пучка труб;

$b$ - относительный продольный шаг пучка труб;

$Q-$ тепловой поток, Вт;

$\lambda$ - коэффициент теплопроводности, Вт/(м·К); 
$\mu$ - динамический коэффициент вязкости, Па·с;

$v$ - кинематический коэффициент вязкости, $\mathrm{M}^{2} / \mathrm{c}$;

$\rho-$ плотность, кг/м ${ }^{3}$.

\section{Безразмерные комплексы:}

$\mathrm{Nu}=\alpha \cdot D$ кан.экв $/ \lambda-$ число Нуссельта;

$\operatorname{Re}=\mathrm{w} \cdot D$ кан.экв $/ v-$ число Рейнольдса;

$\operatorname{Pr}=v / a-$ число Прандтля;

$\mathrm{Eu}=\Delta p / \rho \omega^{2}-$ число Эйлера;

$\Theta=t / t_{\max }$ - безразмерная температура отработанных газов;

\section{Индексы:}

1 - отработанные газы;

2 - вода;

' - теплоноситель на входе;

\section{Введение}

Одним из важнейших направлений энерго- и ресурсосбережения на современном этапе развития техники является разработка более совершенного теплообменного оборудования, входящего в состав энергетических установок. Вместе с тем теплообменные аппараты современных энергетических установок должны отвечать все более ужесточающимся требованиям к новым конструкциям: по надежности работы, минимальному гидравлическому сопротивлению, удобству эксплуатации, компактности, уменьшению массогабаритных показателей и повышению долговечности. Представляет интерес разработка новых усовершенствованных конструкций теплообменников, которые обладают улучшенными энергетическими, массогабаритными и ценовыми характеристиками по сравнению с имеющимися аналогами.

Актуальность исследования, анализ последних исследований и публикаций. Кожухотрубные теплообменные аппараты (ТА), нашли широкое применение в промышленности, энергетике и транспорте, благодаря простоте конструкций, малому гидравлическому сопротивлению, достаточно высокой эффективности и долговечности. Повышение их эффективности напрямую зависит от геометрии и компоновки основного элемента таких теплообменников - трубного
" - теплоноситель на выходе;

$a$ - поперечный ряд труб в пучке;

$b$ - продольный ряд труб в пучке;

кан.экв - эквивалентный межтрубного канала;

кож.экв - эквивалентный кожуха;

in - вход;

cm-стенка;

$s-$ поверхность;

$i=x, y$ индекс для соответственной координаты;

$m$ - локальный;

$c p$ - усредненное значение;

$p$ - изобарный;

$\tau$ - тангенциальный;

$t$ - турбулентный.

пучка. Известно, что для кожухотрубных теплообменников максимальная теплоотдача в трубных пучках наблюдается при перекрестном токе теплоносителей, т. е. при поперечном обтекании таких пучков. Результаты исследований гидродинамики и теплообмена пучков труб (с трубами традиционного круглого сечения), достаточно широко изложены в монографии [1]. Опубликовано значительное количество работ, посвященных исследованиям гладкотрубных пучков с применением труб удобообтекаемой формы [2, 3], а также с различной геометрией и компоновкой в пучках [4-6]. В то же время количество работ посвященных исследованиям процессов гидродинамики и тепло- массопереноса в компактных гладкотрубных пучках, в частности, с трубами малого диаметра, которые образуют ряды с минимальными межтрубными интервалами, весьма незначительно.

Цель работы состоит в численном моделировании и экспериментальном исследовании процессов теплообмена и гидродинамики, протекающих в каналах компактных пучков труб малого диаметра и выполнении их сравнительного анализа с пучками труб известных компоновок.

Экспериментальное оборудование. Для проведения исследований создана экспериментальная когенерационная установка с утилизатором теплоты отработанных газов новой конструкции 
[7], который установлен на линии газовыпускного тракта поршневого двигателя внутреннего

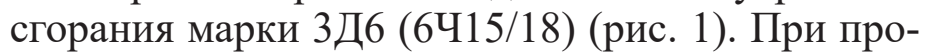
ведении экспериментов сбор и обработка данных измеряемых параметров осуществляется с помощью автоматизированного компьютерного комплекса, в основу которого, положено измерительное оборудование и приспособления предприятий «Регмік» и «Овен».

Поверхность нагрева исследуемого ТА имеет

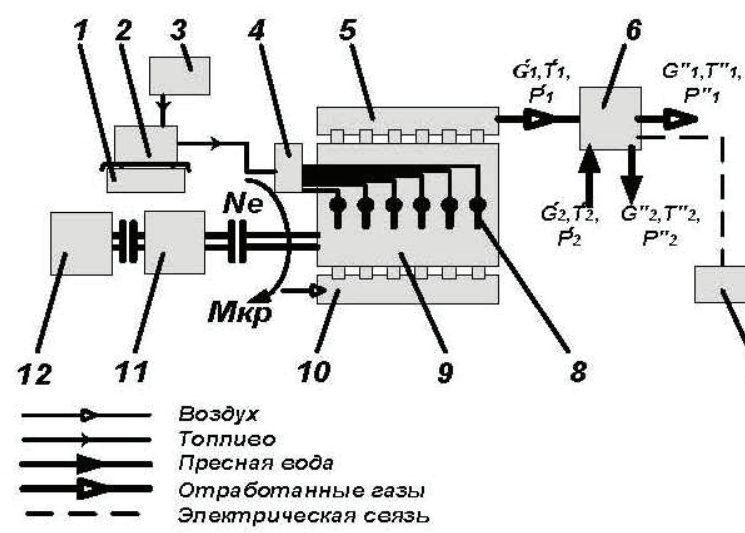

следующие характеристики: диаметр и толщина гладких труб $-d \times \delta=0,010 \times 0,001$ м; эквивалентный диаметр межтрубного канала $-D_{\text {кан.экв }}=9,67$ мм; длина труб $-l=0,150$ м; эквивалентный диаметр кожуха $-D_{\text {кож.экв. }}=0,150 \mathrm{~m}$; суммарная для двух тепловоспринимающих участков поверхность нагрева $-H=1,602$ м

Принципиальная схема и общий вид экспериментальной установки приведены на рис. 1.

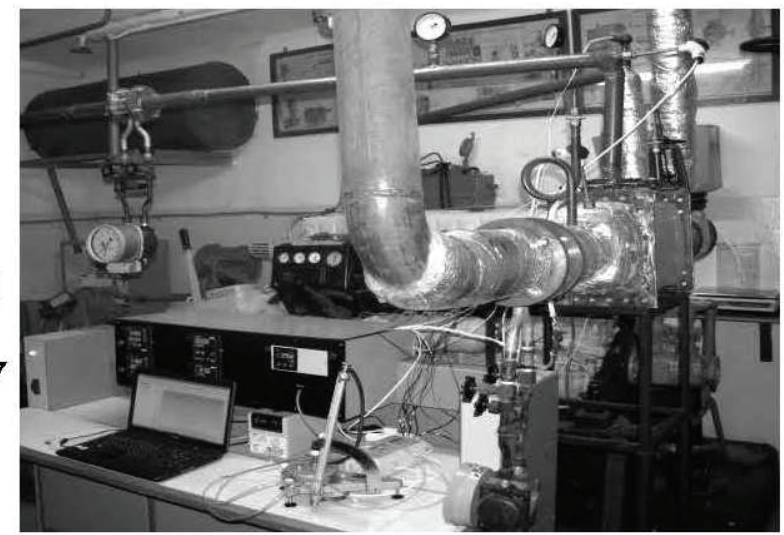

Рис. 1. Принциииальная схема и общий вид экспериментальной установки:

1 - электронные весы; 2 -мерная емкость; 3 - топливный бак;

4 - топливный насос высокого давления (ТНВД); 5 - коллектор отработанных газов;

6 -утилизатор теплоты отработанных газов (ОГ); 7 -автоматизированный компьютерный комплекс измерительного оборудования и приспособлений; 8 - форсунки;

9 - поршневой двигатель внутреннего сгорания; 10 - коллектор впускного воздуха;

11 -реверс-редуктор; 12 - нагрузочное устройство «гидравлический тормоз»;

G, T, P-расход, температура и давление теплоносителей в утилизаторе теплоты.

Экспериментальные исследования для разработанного ТА проводились для пяти режимов работы двигателя при частоте вращения коленчатого вала 600, 800, 1000, 1200 и 1500 об/мин. В результате изменения режимов работы двигателя изменялся расход отработанных газов $G_{1}=0,218 \div 0,328$ кг/с, интервал значений чисел Рейнольдса Re при этом варьировался в диапазоне $3000 \div 6000$. Расход воды выбирался постоянным и был равен $G_{2}=0,175$ кг/с.

\section{Численное моделирование}

Для когенерационной установки на базе двигателя внутреннего сгорания с утилизатором теплоты - ТА новой конструкции проведено компьютерное моделирование процессов гидродинамики и теплообмена, протекающих в каналах исследуемых пучков труб с целью получения локальных распределений поля скоростей, температур и давлений, а также получения зависимостей теплообменных характеристик от динамических и теплофизических параметров теплоносителя, в том числе локальных распределений коэффициентов теплоотдачи по окружности труб 1-4го рядов первого пучка. Геометрия исследуемого канала с компактным расположением пучков труб приведена на рис. 2, который имеет следующие геометрические характеристики: произведение поперечного и продольного шага труб $S_{a} \times S_{b}=0,15 \times 0,10 \mathrm{м}$; диаметр труб $d=0,010 \mathrm{м}$; количество труб в ряду $i=42$ штуки; пучки труб состоят из $j=9$ рядов, а общее количество труб в пучках составляет $z=378$ штук, причем пучки 
разделены между собой технологическим зазором, как показано на рис. 2. Численное моделирование процессов теплообмена и гидродинамики в исследуемых пучках труб проводилось на основе метода конечных элементов с помощью программного пакета ANSYS Fluent. Математическая модель процессов тепло- и массоперено-

$\left.\begin{array}{l}\frac{\partial \rho w_{x}}{\partial \tau}+w_{x} \frac{\partial \rho w_{x}}{\partial x}+w_{y} \frac{\partial \rho w_{x}}{\partial y}=-\frac{\partial p}{\partial x}+\frac{\partial}{\partial x}\left(\mu_{e f} \frac{\partial w_{x}}{\partial x}\right)+\frac{\partial}{\partial y}\left(\mu_{e f} \frac{\partial w_{x}}{\partial y}\right) \\ \frac{\partial \rho w_{y}}{\partial \tau}+w_{x} \frac{\partial \rho w_{y}}{\partial x}+w_{y} \frac{\partial \rho w_{y}}{\partial y}=-\frac{\partial p}{\partial y}+\frac{\partial}{\partial x}\left(\mu_{e f} \frac{\partial w_{y}}{\partial x}\right)+\frac{\partial}{\partial y}\left(\mu_{e f} \frac{\partial w_{y}}{\partial y}\right)\end{array}\right\}$,

ca, которые протекают в исследуемом аппарате при двухмерной (2D) постановке включает в себя систему уравнений Навье-Стокса, уравнение переноса энергии для конвективных течений [8] и стандартную $k$ - $\varepsilon$ модель турбулентности [9]. Уравнения конвективного переноса массы и энергии имеют вид:

$\frac{\partial \rho}{\partial \tau}+\frac{\partial \rho w_{x}}{\partial x}+\frac{\partial \rho w_{y}}{\partial y}=0$

$\frac{\partial t}{\partial \tau}+w_{x} \frac{\partial t}{\partial x}+w_{y} \frac{\partial t}{\partial y}=\frac{1}{\rho c_{p}} \frac{\partial}{\partial x}\left(\lambda_{e f} \frac{\partial t}{\partial x}\right)+\frac{1}{\rho c_{p}} \frac{\partial}{\partial y}\left(\lambda_{e f} \frac{\partial t}{\partial y}\right)$.

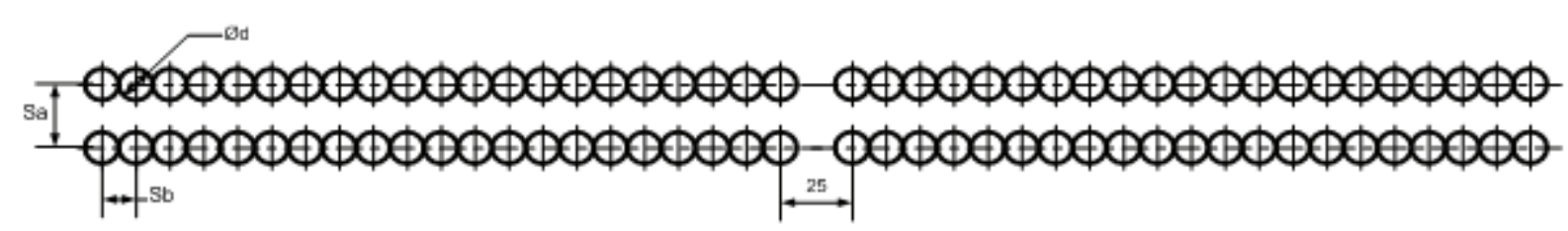

Рис. 2. Геометрия канала с компактным расположением пучка труб.

Для моделирования турбулентного течения используется двухпараметрическая стандартная ных уравнений переноса кинетической энергии и скорости диссипации.

модель, которая состоит из двух дифференциаль-

$\frac{\partial \rho k}{\partial \tau}+w_{x} \frac{\partial \rho k}{\partial x}+w_{y} \frac{\partial \rho k}{\partial y}=\frac{\partial}{\partial x}\left(\frac{\mu_{e f}}{\sigma_{k}} \frac{\partial k}{\partial x}\right)+\frac{\partial}{\partial y}\left(\frac{\mu_{e f}}{\sigma_{k}} \frac{\partial k}{\partial y}\right)+G_{k}-\rho \varepsilon$,

$\left.\frac{\partial \rho \varepsilon}{\partial \tau}\right)+w_{x} \frac{\partial \rho \varepsilon}{\partial x}+w_{y} \frac{\partial \rho \varepsilon}{\partial y}=\frac{\partial}{\partial x}\left(\frac{\mu_{e f}}{\sigma_{\varepsilon}} \frac{\partial \varepsilon}{\partial x}\right)+\frac{\partial}{\partial y}\left(\frac{\mu_{e f}}{\sigma_{\varepsilon}} \frac{\partial \varepsilon}{\partial y}\right)+c_{\varepsilon 1} G_{k} \frac{\varepsilon}{k}-c_{\varepsilon 2} \rho \frac{\varepsilon^{2}}{k}$,

$\partial e \mu_{e f}=\mu+\mu_{t}, \lambda_{e f}=\lambda+\lambda_{t}, \mu_{t}=\frac{c_{\mu} \rho k^{2}}{\varepsilon}, G_{k}=\mu+\frac{\partial w_{x}}{\partial x_{i}}\left(\frac{\partial w_{j}}{\partial x_{i}}+\frac{\partial w_{i}}{\partial x_{j}}\right)$,

$c_{\mu}=0.09, c_{\varepsilon 1}=1.44, c_{\varepsilon 2}=1.92, \sigma_{k}=1, \sigma_{\varepsilon}=1.3$.

Краевые условия имеют вид $w_{i}(\tau=0)=0, w_{i}(x=0)=w_{i n}, w_{i}\left(x_{i}=x_{\mathrm{CT}}\right)=0,\left.t\right|_{s}=\left.t_{C T}\right|_{s}$.

Численные расчеты в исследуемых пучках труб проведены для пяти режимов работы двигателя при частоте вращения коленчатого вала $n 600$, 800, 1000, 1200 и 1500 об/мин. Результаты расчетов для режима работы 1500 об/мин приведены на рис. 3, a-c. Распределение поля скоростей в каналах трубных пучков представлено на рис. 3а. Значение средней скорости ОГ в наиболее узком поперечном сечении канала составляет $372 \mathrm{~m} / \mathrm{c}$ (рис. $3, a)$. На рис. $3, b$ и 3 , с показано температурные распределения и изменение давления в потоке ОГ, протекающих по каналам трубных пучков. 

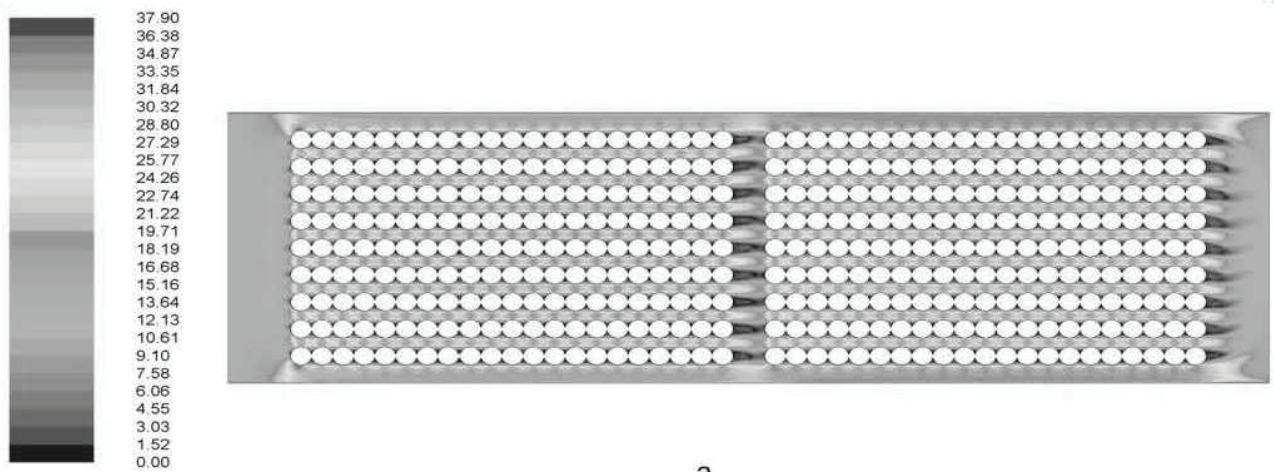

Contours of Velocity Magnitude ( $\mathrm{m} / \mathrm{s})$
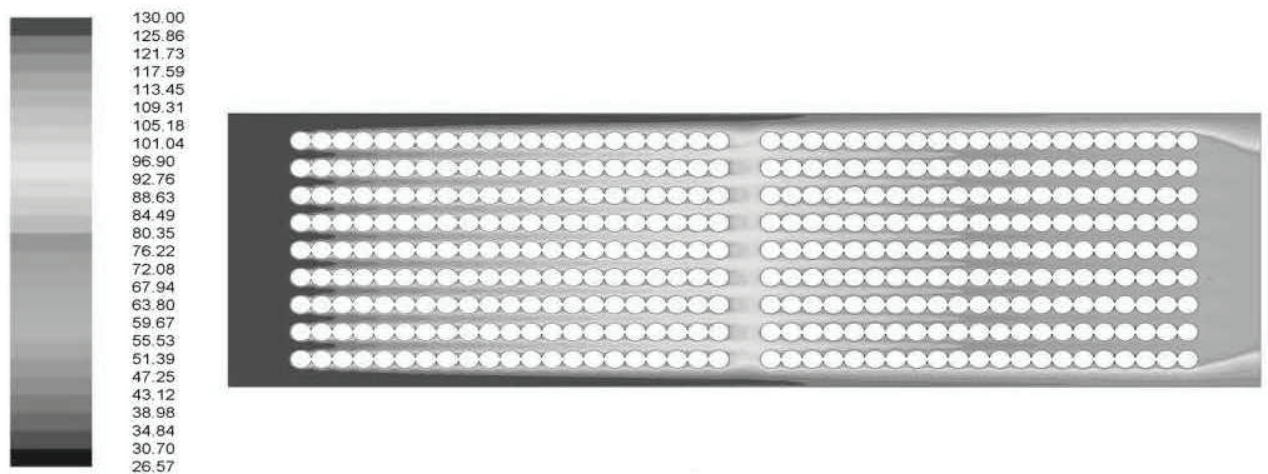

b

Contours of Total Temperature (c)
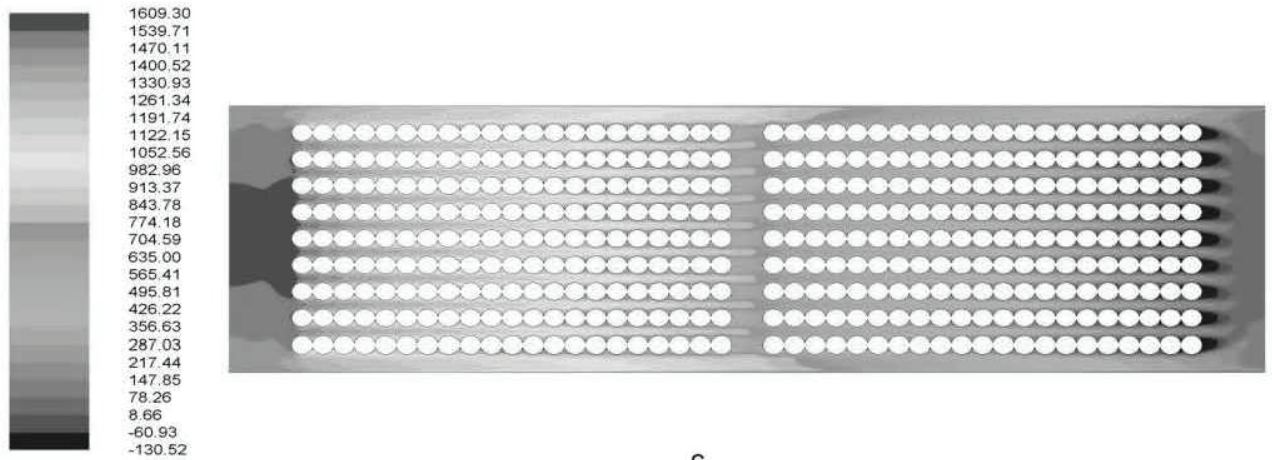

$-130.52$

c

Contours of Total Pressure (pascal)

Рис. 3. а-скорость отработанных газов в каналах пучков труб, м/с;

b-изменение температуры в каналах пучков труб, ${ }^{\circ} \mathrm{C}$; c-перепад давления в каналах пучков труб, Па.

Как видно из рисунка 3, $b$ газы, выходящие из боковых каналов между корпусом и пучками, обладают более высокими температурами по сравнению с газами, выходящими из каналов пучка, что указывает на наличие байпасной протечки газов между трубным пучком и корпусом аппарата.
В результате численного моделирования получены локальные распределения коэффициента теплоотдачи по окружности труб 1-4-го поперечных рядов первого пучка для каждого из пяти режимов работы двигателя (рис. 4 для $n=1500$ об/ мин). 


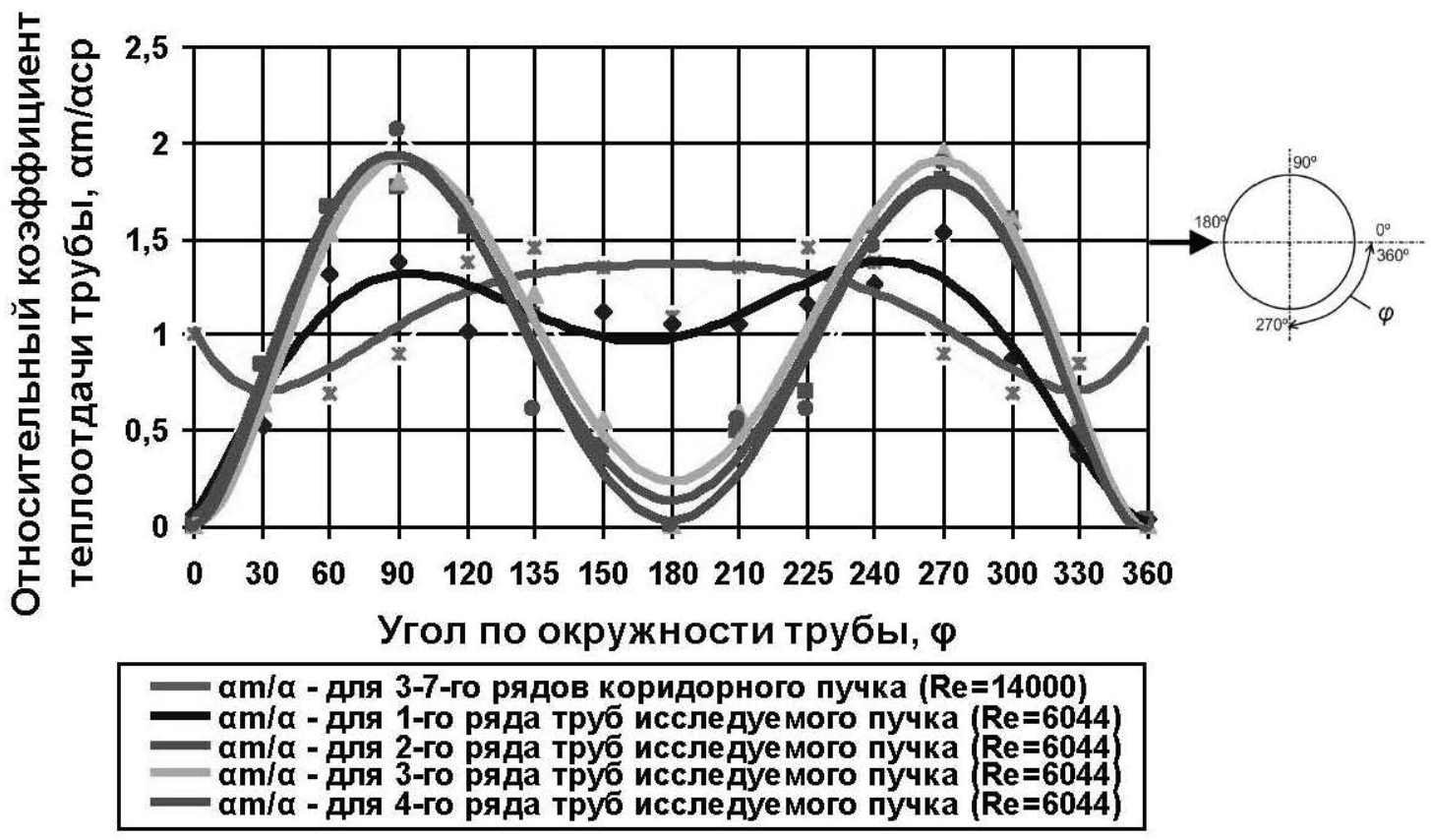

Рис. 4. Распределение локальных коэффициентов теплоотдачи $\alpha_{m} / \alpha_{c p}$ на поверхности первых четырех труб продольного ряда исследуемого пучка с относительным поперечным и продольным шагом $a \times b=1,5 \times 1,0$ при $\operatorname{Re}=6044 ; \operatorname{Pr}=0,692(n=1500$ об/мин) и аппроксимирующей полиномиальной функции распределения относительного коэффициента теплоотдачи $\alpha_{m} / \alpha_{c p}$ на поверхности труб 3-7-го рядов коридорного пучка при Re=14000 [10].

Анализ результатов исследований. Сравнение интенсивности теплоотдачи для труб 1-4-го поперечных рядов первого пучка для пяти установленных режимов работы двигателя проводились на основе полученных численных данных о локальных распределениях коэффициентов теплоотдачи на их поверхности.

На рис. 4 показано распределение локальных значений коэффициента теплоотдачи по периметру для труб 1-4-го ряда в направлении потока (первый трубный пучок), представленных в безразмерной форме $\alpha_{m} / \alpha_{c p}$, где $\alpha_{c p}-$ усредненное значение коэффициента теплоотдачи по периметру трубы. Локальные распределения, получены для каждого из пяти режимов работы двигателя и, кроме того, выполнено сравнение полученных результатов с результатами известных исследований для коридорного расположения трубных пучков.

Анализ полученных распределений показывает, что локальные максимумы коэффициента теплоотдачи наблюдаются для труб 1-го ряда вблизи лобовой точки натекания потока, а для труб 2-4 рядов в областях присоединения потока к поверхности труб. В области отрывных течений локальные значения $\alpha_{m} / \alpha_{c p}$ существенно меньше, что обусловлено небольшими значениями скорости потока в отрывных зонах и наличием застойных зон гидродинамического течения.

Сравнение локальных значений коэффициента теплоотдачи для исследуемого компактного пучка труб и пучка с коридорным их расположением показывает, что при компактном расположении труб величина и количество локальных максимумов больше по сравнению с коридорным их расположением, что в конечном итоге приводит к улучшению интегральных теплообменных характеристик пучка.

Интенсификация теплообмена в пучках с компактным расположением труб по сравнению с коридорным пучком достигается влиянием на условия теплообмена нескольких факторов: 1) уменьшением диаметра труб, что приводит к сокращению длины зон формирования пограничного слоя на поверхности труб в области присоединения внешнего потока к их поверхности; 2) уменьшением межтрубного расстояния (шага) между соседними рядами труб, что приводит к 
увеличению скорости внешнего потока при одинаковом расходе теплоносителя в теплообменных аппаратах известных конструкций; 3) большей турбулизацией внешнего потока в межтрубных каналах, что интенсифицирует теплообмен на обтекаемой поверхности; 4) созданием продольных знакопеременных градиентов давления, что также приводит к интенсификации теплообменных процессов (аналог каналов с переменным сечением или «гофрированных» каналов типа диффузор-конфузор).

Для исследуемых пучков значение коэффициента теплоотдачи $\alpha_{c p}$ на участках присоединенного течения существенно больше значений $\alpha_{c p}$ на участках отрывного течения. При этом максимальное значение относительного коэффициента теплоотдачи 2-4-го рядов труб $\alpha_{m} / \alpha_{c p}$ на участках присоединенного течения (при $\mathrm{Re}=6044)$ превышает на 0,46 (29\%) максимальное значение $\alpha_{m} / \alpha_{c p}$ для 3-7-го рядов труб коридорного пучка (при $\mathrm{Re}=14000)$. Причем у исследуемого пучка максимумов $\alpha_{m} / \alpha_{c p}$ больше вдвое, которое обусловлено уменьшением длины формирования пограничного слоя на трубах малого диаметра, что в конечном результате повышает интенсивность процесса теплообмена. Этот фактор конструктивно обеспечивается «гофрированностью» канала, т. е. последовательным чередованием участков сужения и расширения вдоль канала. При этом относительный коэффициент теплоотдачи выше в местах сужения канала, чем в местах его расширения.

Течение и теплообмен в разработанных пучках организуется следующим образом. При поперечном обтекании рядов труб возникают отрывные течения в угловых зонам между соседними трубами. Оторвавшийся от поверхности цилиндра поток, который формируется между точками поверхности при угле по окружности трубы $\varphi=75^{\circ}$ и $\varphi=$ $290^{\circ}$, присоединяется к поверхности низлежащего цилиндра в точках при угле по окружности трубы $\varphi=110^{\circ}$ и $\varphi=255^{\circ}$ (рис. $3, a$ ). Возникшая застойная зона между трубами испытывает периодические воздействия поперечных пульсаций давления. Из этой области периодически, с определенной частотой, выбрасывается масса теплоносителя, взаимодействие которой с основным потоком приводит к турбулизации течения.
При наличии соприкосновения соседних труб, которое используется в компоновке компактного пучка, как известно на примере двух соприкасающихся труб (тандеме) $[1,11,12]$ существенно уменьшает аэродинамическое сопротивление трубного пучка, поскольку расположенные один за другим цилиндры обтекаются без возникновения крупномасштабных вихрей, которые образуются, например, при обтекании труб с коридорным расположением.

Результаты исследований течения и теплообмена в пучках труб ТА новой конструкции оценивались путем сравнения полученных экспериментальных данных с результатами численного моделирования и известными экспериментальными данными, которые получены другими исследователями. Результаты исследований представлены в виде графических зависимостей на рис. 5-7 для чисел Рейнольдса в диапазоне $\mathrm{Re}=3000 \div 6000$ и числа Прандтля $\operatorname{Pr}=0,73$.

На рис. 5 приведены зависимость безразмерной температуры отработанных газов $\theta(a)$ и безразмерного количества теплоты $Q / Q_{\max }(b)$ от частоты вращения коленчатого вала двигателя $n$.

На рис. 6, $a$ и 6, $b$ представлены зависимости числа Эйлера и перепада давления отработанных газов от числа Рейнольдса.

На рис. 7, $а$ представлена зависимость усредненного по поверхности коэффициента $\bar{\alpha}$ теплоотдачи от числа Рейнольдса Re. Как следует из рисунка, возрастание скорости потока отработанных газов в 2 раза приводит к росту $\bar{\alpha}$ в 1,8 раза. Сопоставление расчетных и экспериментальных данных показывает, что погрешность, полученная при численном моделировании, не превышает $5 \%$.

На рис. 7, $b$ приведена экспериментально найденная зависимость усредненного по поверхности числа Нуссельта $\bar{N} u$ от числа Рейнольдса Re. Как видно из рисунка, для полученной зависимости в области больших чисел Рейнольдса наблюдается более быстрый рост числа Нуссельта. На рис. 7 приведена также экспериментальная зависимость $\bar{N} u=f(\mathrm{Re})$, полученная для коридорного пучка труб в работах А.А. Жукаускаса, И.А. Белова и Н.А. Кудрявцева $[1,11]$. Сопоставление показывает, что при тех же значениях числа Рейнольдса интенсивность теплообмена для 
Q/Qmax

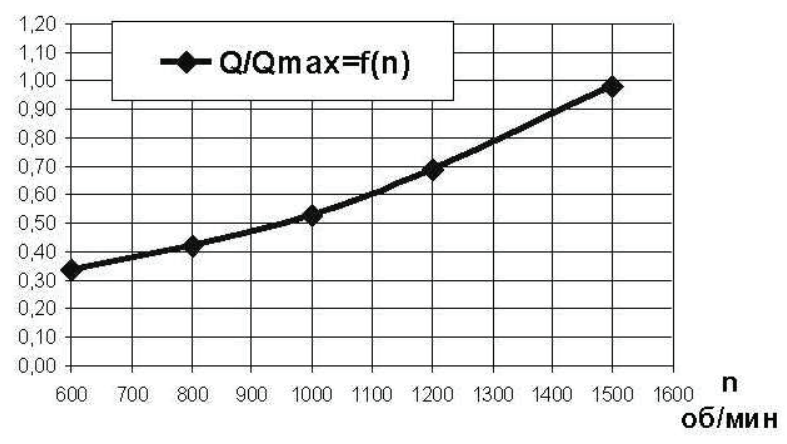

$\theta$

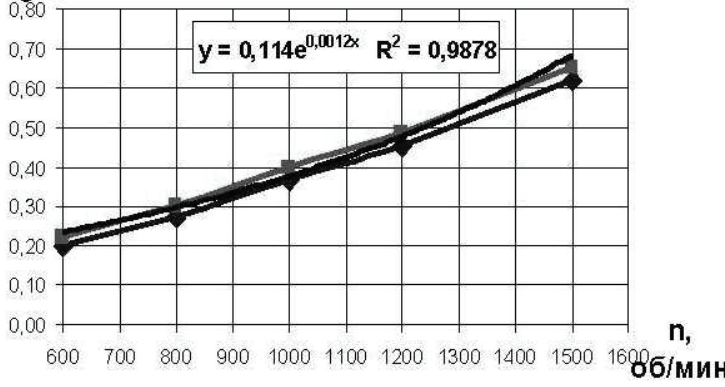

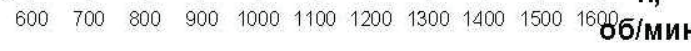

$\neg \theta=f(n)-$ экспериментальные данные для компактного пучка труб $-\theta=f(n)-$ результаты численного моделирования

- Аппроксимирующая экспоненциальная кривая $\theta=f(n)$

\section{a)}

\section{b)}

Рис. 5. Зависимость безразмерной температуры отработанных газов $\theta$ (а) и безразмерного количества теплоты $Q / Q_{\text {max }}$ (b) от частоты врацения коленчатого вала двигателя n:

- экспериментальные данные для компактного пучка труб;

- результаты численного моделирования.

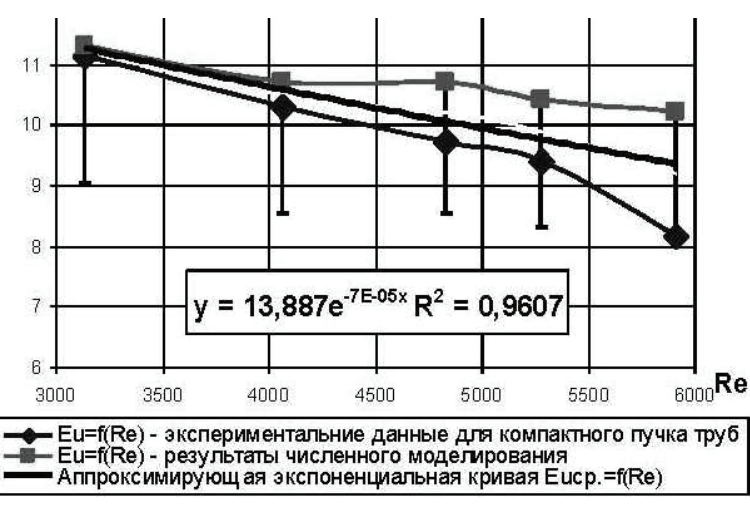

a)

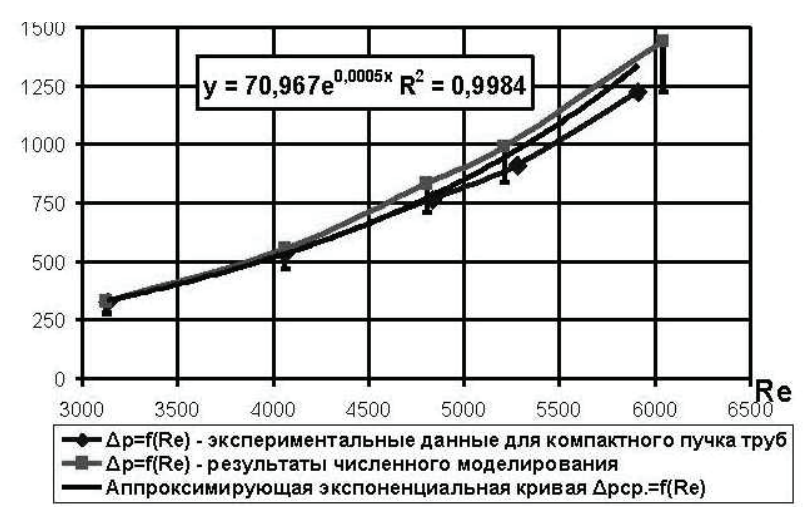

b)

Рис. 6. Зависимость числа Эйлера Еи (a) и перепада давления Ар (b) отработанных газов от числа Рейнольдса Re: - экспериментальные данные для компактного пучка труб;

- результаты численного моделирования.

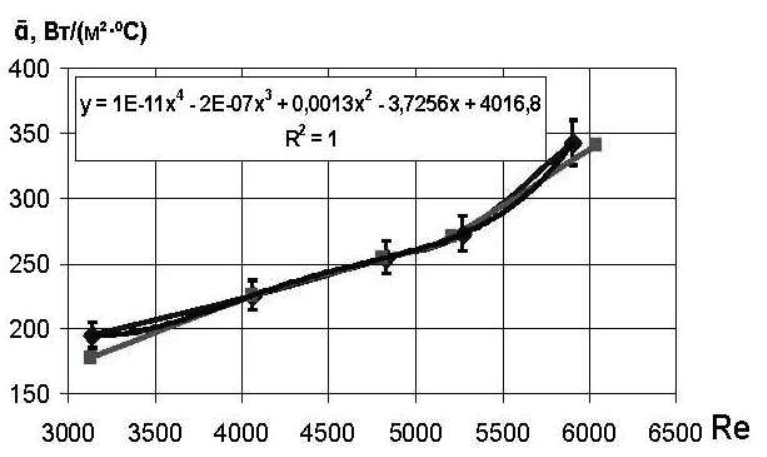

- $\overline{\mathrm{a}}=\mathrm{f}(\mathrm{Re})$ - экспериментальные данные для компактного пучка труб - $-\overline{\mathrm{a}}=\mathrm{f}(\mathrm{Re})$ - результаты численного моделирования - Аппроксимирующая полиномиальная кривая $\bar{a}=f(R e)$

a)

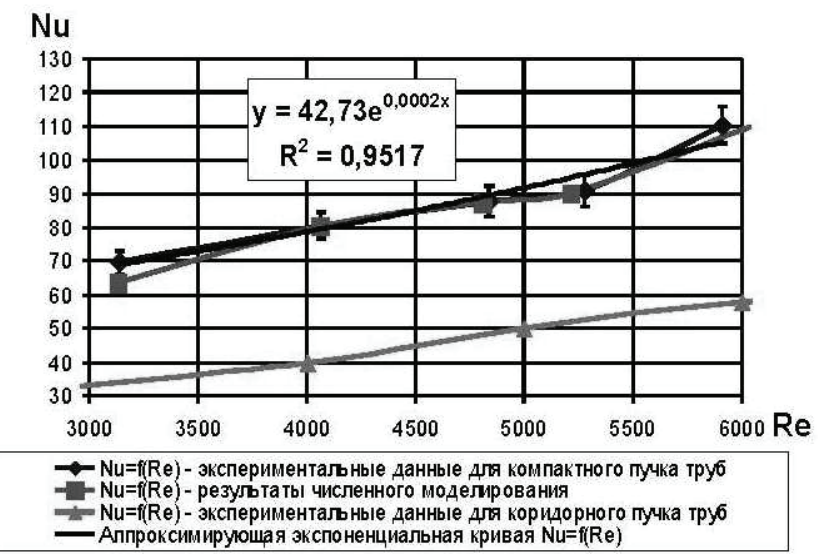

b)

Рис. 7. Зависимость усредненного по поверхности коэффициента теплоотдачи $\bar{\alpha}$ (а) и среднего числа Нуссельта $\bar{N}$ (b) от числа Рейнольдса Re: - экспериментальные данные для компактного пучка труб; А- экспериментальные данные для коридорного пучка труб;

- результаты численного моделирования. 
компактного пучка труб малого диаметра почти в 2 раза превышает интенсивность теплообмена на поверхности коридорного пучка труб.

Исходя из результатов экспериментальных исследований показано, что предложенная конструкция ТА с компактным трубным пучком имеет высокую эффективность, которая существенно превышает тепловую эффективность кожухотрубного теплообменника с традиционной коридорной компоновкой труб. Расчеты показывают, что теплообменные аппараты новой конструкции имеют габаритные размеры на $50 \%$ и массу на $10 \%$ меньше по сравнению с теплообменными аппаратами известных конструкций.

\section{Выводы}

1. Проведено компьютерное моделирование процессов тепло- и массопереноса в каналах предельно сжатых поперечно обтекаемых гладкотрубных пучков коридорной компоновки при отсутствии зазора между соседними трубами в направлении движения теплоносителя при помощи программного комплекса ANSYS Fluent. Получены поля скоростей, температур и давлений в исследуемых каналах, проанализировано условия гидродинамического течения в них и проведена оценка интенсивности теплопереноса между теплоносителями через стенку, которая их разделяет.

2. Получены экспериментальные данные процессов, протекающих в каналах исследуемого пучка, которые представлены в виде зависимостей теплообменных характеристик для сжатых пучков труб от динамических и теплофизических параметров теплоносителей.

3. Определено, что максимальное значение относительного коэффициента теплоотдачи на поверхности труб 2-4-го рядов $\alpha_{m} / \alpha_{c p}$ на участках присоединенного течения (при $\mathrm{Re} \stackrel{c p}{=} 6044)$ превышает на 0,46 (29 \%) максимальное значение $\alpha_{m} / \alpha_{c p}$ для труб 3-7-го рядов при коридорной компоновке пучков (при $\mathrm{Re}=14000)$. Показано, что у исследуемого пучка максимумов $\alpha_{m} / \alpha_{c p}$ примерно в 2 раза больше по сравнению с коридорным пучком, что обуславливает увеличение суммарного коэффициента теплообмена для пучков новой конфигурации.

4. Проведен сравнительный анализ результатов численного моделирования и экспери- ментальных данных для каналов с компактным размещением пучков труб. Показано, что разработанная конструкция является достаточно эффективной при относительно малом гидравлическом сопротивлении и имеет улучшенные массогабаритные показатели теплообменной поверхности при одинаковой тепловой мощности теплообменников.

5. Предложена компактная конструкция теплообменного трубного пучка, которая обладает высокой эффективностью, малым аэродинамическим (1225 Па) и гидравлическим сопротивлением (400 Па), при значениях числа Нуссельта на поверхности исследуемого пучка, которые примерно в 2 раза превышают их значения для пучка с коридорной компоновкой труб.

\section{ЛИТЕРАТУРА}

1. Жукаускас A.A. Конвективный перенос в теплообменниках. - Москва: Наука, 1982. - 472 с.

2. Письменный Е.Н., Кондратюк В.А., Жукова Ю.B., Терех A.M. Конвективный теплообмен поперечно-омываемых шахматных пакетов плоскоовальных труб // Восточно-европейский журнал передовых технологий. - 2011.-2/8 (50). - С. 4-8.

3. Pis'mennyi, E.N. Ways for Improving the Tubular Heaters Used in Gas Turbine Units // Thermal Engineering. - 2012. - Vol. 59, Issue 6. P. 485-490.

4. Анисин А.А. Повышение энергетической эффективности пучков гладких труб и профилированных каналов для газо-жидкостных теплообменных аппаратов энергетических установок: автореферат диссертации на соискание степени доктора техн. наук: 05.14.04 /А.А. Анисин; Санкт-Петербургский государственный политехнический университет. - СПб.: 2009. - 42 с.

5. Пронин В.А., Дозориев А.В., Тырин В.E. Гидродинамика и теплообмен в межтрубных каналах гладкотрубных пучков коридорно-диффузорного типа // Труды четвертой Российской Национальной конференции по теплообмену: В 8 томах. Т.6. Дисперсные потоки и пористые среды. Интенсификация теплообмена. - М., 2006. C. $285-288$.

6. Пронин B.A. Компоновки трубных пучков и синтез конвективных поверхностей теплообмена 
с повышенной энергоэффективностью: автореферат диссертации на соискание степени доктора техн. наук: 01.04.14 / В.А. Пронин; Московский энергетический институт. - М.: 2008. - 40 с.

7. Патент UA 104559, F28D 7/00 (2014.01). Теплообмінний апарат / Горобець В.Г., Богдан Ю.О.; заявник Горобець В.Г., Богдан Ю.О. - № а 201303816; заявл. 27.03.2013; опубл. 10.02.2014, Бюл. №3, 2014 р. -2 с: іл..

8. Шлихтинг Г. Теория пограничного слоя. М.: Наука, 1974. - 712 с.
9. ANSYS FLUENT Theory Guide. Release 14. ANSYS, Inc. Southpointe 275 Technology Drive Canonsburg, PA 15317, 2011. - 826 p.

10. Кулинченко В.Р. Справочник по теплообменным расчетам. - К.: Тэхника, 1990. - 165 с.

11. Белов И.А., Кудрявцев Н.А. Теплопередача и сопротивление пакетов труб. - Л.: Энергоатомиздат, $-1987 .-223$ с.

12. Идельчик И.Е. Справочник по гидравлическим сопротивлениям. - М.: Машиностроение, 1992. -672 c. 


\section{NUMERICAL MODELING AND EXPERIMENTAL INVESTIGATION OF HEAT EXCHANGE AND HYDRODYNAMICS PROCESS IN HEAT EXCHANGER OF NEW DESIGN}

\section{Gorobets V.G. ${ }^{1}$, Bohdan Yu.O. ${ }^{2}$, Trohanyak V.I. ${ }^{1}$}

${ }^{1}$ National University of Life and Environmental Sciences of Ukraine, Heroyiv Oborony st., 15, Kyiv, 03041, Ukraine

${ }^{2}$ Kyiv State Maritime Academy named after hetman Petro Konashevich-Sahaydachniy, Kyrilivska str., 9, Kyiv, 04071, Ukraine

In this paper, the computer numerical modeling and experimental investigation of heat and hydrodynamic characteristics of compressed round tube bundle for heat exchangers of new design are presented. Comparative analysis of obtained results with the results of known experimental investigation for smooth tube banks of inline arrangements are realized. Comparison of the results of numerical modeling of compressed round tube bundle with results of finished experiment and known experimental data for round tube bundle of convention inline geometry, confirms the adequacy of modeling and almost double the increase in the thermal efficiency of the investigated compressed round tube bundle with a relatively small change in the total aerodynamic resistance.

References 12, fig. 7.

Key words: heat exchange, hydrodynamics, tube bundle, numerical modeling, experiment

1. Zhukauskas A.A. Convective heat transfer in heat exchangers. - Moscow: Nauka, 1982. - 472 p. (Rus.)

2. Pis'mennyi, E.N., Kondratyk V.A., Zhukova $Y$ u.V., Tereh A.M. Convective heat exchange of crosswashing straggle packs of flat-oval tubes // EasternEuropean Journal of Enterprise Technologies. -
2011. - 2/8 (50). - P. 4-8. (Rus.)

3. Pis'mennyi, E.N. Ways for Improving the Tubular Heaters Used in Gas Turbine Units // Thermal Engineering. - 2012. - Vol. 59, Issue 6. - P. 485-490.

4. Anisin A.A. Increase of energy efficiency of smooth tube bundles and profile channels for gasliquid heat exchangers for power plants: abstract of thesis for the degree of doctor of technical science on the speciality: 05.14.04 /A.A. Anisin; Peter the Great Saint-Petersburg Polytechnic University. - SaintPetersburg: 2009. - 42 p. (Rus.)

5. Pronin V.A., Dozortsev A.V., Tyirin V.E. Hydrodynamic and heat transfer in tube channels of smooth tube bundles of inline-diffuser type // Transactions of the fourth Russian National heat exchange conference: In 8th volumes. Vol.6. Dispersed flows and porous mediums. Heat exchange intensification. - Moscow, 2006. - P. 285-288. (Rus.)

6. Pronin V.A. Arrangements of tube bundles and synthesis of convective heat surfaces with increased energy efficiency: abstract of thesis for the degree of doctor of technical science on the speciality: 01.04.14 / V.A. Pronin; Moscow Power Engineering Institute. - M.: 2008. - 40 p. (Rus.)

7. Patent UA 104559, F28D 7/00 (2014.01). Heat exchanger / Horobets' V.H., Bohdan Yu.O.; zayavnyk Horobets' V.H., Bohdan Yu.O. - № a 201303816; zayavl. 27.03.2013; opubl. 10.02.2014, Byul. №3, 2014 year. -2 p: il.. (Ukr.)

9. ANSYS FLUENT Theory Guide. Release 14. ANSYS, Inc. Southpointe 275 Technology Drive Canonsburg, PA 15317, 2011. - 826 p.

10. Kulinchenko V.R. Reference book to heat transfer calculation. - K.: Tehnika, 1990. - 165 p. (Rus.)

11. Belov I.A., Kudryavtsev N.A. Heat transfer and resistance of tube packs. - Leningrad: Energoatomizdat, - 1987. - 223 p. (Rus.)

12. Idel'chik I.E. Reference book to hydraulic resistance. - M.: Mashinostroenie, 1992. - 672 p. (Rus.) 